\title{
Associated Study
}

National Cancer Institute

\section{Source}

National Cancer Institute. Associated Study. NCI Thesaurus. Code C90359.

An indication that one study is related to another. 